workers stopped, when the research will gradually die out from want of support. On the other hand, if, as you, Sir, admit, we are on a track of a discovery which will in time enable us to foretell the cycle of droughts, public opinion should demand that the investigation be prosecuted with redoubled vigour and under better conditions.

My object in writing this letter is to express a hope that your powerful influence may not be wanting in furtherance of a branch of inquiry from which I, as an individual worker in it, am undoubtedly of opinion that the greatest national benefit must in time arise. If forewarned be forearmed, then such a research will ultimately conduce to the saving of life both at times of maximum and minimum sun-spot frequency.

Owens College, Manchester, September 20

\section{PROF. ADAMS ON LEVERRIER'S PLANETARY THEORIES}

THE following admirable statement concerning Le1 verrier's more recent work was made on the occasion Society, in February, last year, to him by Prof. J. C. Adams, the president. It will be read with a mournful interest at the present time :-

It is not many years since our medal was awarded to M. Leverrier for his theories and tables of the four planets nearest the sun, viz., Mercury, Venus, the Earth, and Mars. Long before this he had been occupied with the larger planets, but before proceeding further with their theories he found it necessary to establish on solid foundations the theory of the motion of the earth, on which all the rest depend, and this again naturally led him to investigate the theories of the three nearer planets which, with the earth, constitute the inferior portion of the plinetary system.

By the comparison of these theories with observations, II. Leverrier was led to two interestirg results. He found that in order to bring the theories of Mercury and Mars into accordance with observation, it was neces sary and sufficient to increase the secular motion of the perihelion of Mercury, and also the secular motion of the perihelion of Mars.

Hence M. Leverrier inferred that there existed on the one hand, in the neighbourhood of Mercury, and on the other, in the neighbourhood of Mars, sensible quantities of matter the action of which had not been taken into account.

This conclusion has been verified with respect to Mars. The matter which had not been considered, turns out to belong to the earth itself, the mass of which had been taken too small, having been derived from too small a value of the solar parallax. A similar increase of the mass of the earth is indicated by the theory of Venus, and a corresponding increase of the solar parallax is likewise derived from the lunar equation in the motion of the sun.

With respect to Mercury, a similar verification has not yet taken place, but the theory of the planet has been established with so much care, and the transits of the planet across the sun furnish such accurate observations, as to leave no doubt of the reality of the phenomenon in question; and the only way of accounting for it appears to be to suppose, with M. Leverrier, the existence of several minute planets, or of a certain quantity of diffused matter circulating about the sun within the orbit of Mercury.

The results which $M$. Leverrier had thus obtained from his researches on the motions of the inferior planets added to the interest with which he now entered upon similar researches on the system of the four great planets which are the most distant from the sun. Such researches might furnish information respecting matter hitherto un. known existing in the neighbourhood of these planets.
Possibly they might afford indications of the existence of a planet beyond Neptune, and at any rate they would provide materials which would facilitate future discoveries.

As I shall have occasion to explain later on, the theories of the mutual disturbances of the larger planets are far longer and more complicated than those of the smaller, so that all that $M$. Leverrier had yet done might be almost regarded as merely a prelude to what still remained to be done. Increased difficulties, however, far from deterring, seemed rather to stimulate him to greater exertions.

On May 20, I872, M. Leverrier presented to the Academy an elaborate memoir, containing the first part of his researches on the theories of the four superior planets, Jupiter, Saturn, Uranus, and Neptune. This memoir contains an investigation of the disturbances which each of these planets suffers from the action of the remaining three. Throughout this investigation the development of the disturbing function, as well as that of the inequalities of the elements is given in an algebraical form, in which everything which varies with the time is represented by a general symbol, so that the expressions obtained hold good for any time whatever. Thus the eccentricities and inclinations, the longitudes of the perihelion and of the nodes are all left in the condition of variables. The mean parts of the major axes, which suffer no secular variations, are alone treated as given numbers.

At the end of the résumé of the contents of this memoir, given in the Comptes Rentus, M. Leverrier lays down the following almost appalling programme of the work still remaining to be done.

It would be necessary, he says, -

I. To calculate the formulie, and to reduce them into provisional tables.

2. To collect all the exact observations of the four planets, and to discuss them afresh, in order to refer their positions to ore and the same system of co-ordinates.

3. By means of the provisional tables, to calculate the apparent positions of the planets for the epochs of the observations.

4. To compare the observed with the calculated positions, to deduce the corrections of the elliptic elements of the four planets, and to examine whether the agreement is then perfect.

5. In the contrary case, to find the causes of the discrepancy between theory and observation.

Extensive as is this programme, it has already been completely carried out as regards the planets Jupiter and Saturn, and partly so as regards Uranus and Neptune.

Having received from the Academy the most effectual encouragement to pursue his researches, M. Leverrier lost no time in bringing them gradually to completion, so that they might become available for practical use.

Accordingly, on August 26, 1872 , he presented to the Academy a memoir containing a complete determination of the mutual disturbances of Jupiter and Saturn, and thus serving as a base for the theories of both these planets, which are closely connected with each other.

Again, on November I I, 1872, he presented his determination of the secular variations of the elements of the orbits of the four planets, Jupiter, Saturn, Uranus, and Neptune. These variations are mutually dependent on each other, and must be treated simultaneously. Their determination consequently involves the solution of sixteen differential equations, which are very complicated in form, and can only be integrated by repeated approximation.

This part of the work forms a necessary preliminary to the treatment of the theory of any one of these planets in particular.

On March 17, 1873, M. Leverrier presented to the Academy the complete theory of Jupiter ; and on July I4 in the same year he followed it up by the complete theory of Saturn. 
On January 12,1874 , he presented his tables of Jupiter, founded on the theory which has just been mentioned, as compared with observations made at Greenwich from 1750 to 1830 , and from 1836 to 1869 , and with observations made at Paris from 1837 to 1867 .

Again, on November 9, I874, he presented to the Academy a complete theory of Uranus. Already in $r 846$, in his researches which led to the discovery of Neptune, $M$. Leverrier had given a very full investigation of the perturbations of Uranus by the action of Jupiter and Saturn. In the memoir just mentioned he gives a fresh investigation, including a full treatment of the perturbations of Uranus by the action of Neptune.

On December 14,1874 , he presented a new theory of the planet Neptune, thus completing the theoretical part of the immense labours which he had undertaken with respect to the planetary system.

Finally, on August 23,1875 , he presented to the Academy the comparison of the theory of Saturn with observations.

Such is a bare enumeration of the various labours for which our science is already indebted to our illustrious Associate.

That any one man should have had the power and perseverance required thus to traverse the entire solar system with a firm step, and to determine with the utmost accuracy the mutual disturbances of all the primary planets which appear to have any sensible influence on each other's motions, might well have appeared incredible if we had not seen it actually accomplished.

I will now proceed to give a brief outline of the investigations relating to the motions of the four larger planets, with which we are now more particularly concerned. The most important parts of these investigations are printed in full detail in the volumes of Memoirs which form part of the Amnals of the Observatory of Paris.

As in his former rescarches, $M$. I.everrier here also exclusively employs the method of variation of elements, and the investiotions are based on the development of the disturbing function given by him, in the first volume of the Annals of the Paris Observatory, with greater accuracy and to a far greater extent than had ever been done before.

The eighteenth chapter of M. Leverrier's researches, which forms nearly the whole of the tenth volume of the Memoirs, is devoted to the determination of the mutual action of Jupiter and Saturn, which forms the foundation of the theories of these two planets.

These theories are extremely complicated, and I shall endeavour briefly to point out, and to explain as far as I can without the introduction of algebraical symbols, the nature of the peculiar difficulties which M. Leverrier has nature of the pecuilar dir encounter in their treatment, and which he has so successfully overcome. These difficulties either do not present themselves at all, or do so in a very minor degree in the theories of the smaller planets.

First, then, the masses of Jupiter and Saturn are far larger than those of the inferior planets, the mass of Jupiter being more than 300 times and that of Saturn being nearly 100 times greater than the mass of the eartb. For this reason it is necessary to develop the infinite series in which the perturbations are expressed to a much greater extent when we are dealing with Jupiter and greater extent when when we are concerned with the mutual disturbances of the inferior planets. Also Jupiter and Saturn are so far removed from these latter planets that the disturbances which they produce in the motion of these planets are extremely small, in spite of the large masses of the disturbing bodies.

But the great magnitude of the disturbing masses is far from being the only reason why the theory of the mutual disturbances of Jupiter and Saturn is so complicated.

Another cause which aggravates the effect of the former is the near approach to commensurability in the mean motions.

Twice the mean motion of Jupiter differs very little from five times that of Saturn. In other words, five periods of Jupiter occupy nearly the same time as two of Saturn, so that if at a given time the planets were in conjunction at certain points in their orbits, then after three synodic periods they would be again in conjunction at points not far removed from their positions at starting. Hence, whatever uncompensated perturbations may have been produced in the motions of the two planets during these three synodic periods will be very nearly repeated in the next three synodic periods, and again in the next three, and so on.

Hence the disturbances will go on accumulating in the same direction during many revolutions of the two planets, and will become very important. The inequalities of long period thus arising will affect all the elements of the orbits of the two planets; but the most important are those which affect the mean longitudes of the bodies, since these are proportional to the square of the period of the inequalities, whereas the inequalities affecting the other elements are proportional to the period itself.

The principal terms of the inequalities of mean longitude are of the third order, if we consider the excentricities of the orbits and their mutual inclination to be small quantities of the first order.

Terms of the same period, however, and those far more numerous and more complicated in expression, occur among those of the fifth and of the seventh order of small quantities, and M. Leverrier has included these terms also in his approximations.

But the circumstance which contributes in the highest degree to cause the superior complexity of the theories of the larger planets is the necessity, in their case, of taking into account the terms which depend on the squares and higher powers of the disturbing forces.

I will endeavour to point out the nature of these terms and the manner in which they arise.

By the theory of the variation of elements we are able to express at any given time the rate of variation of any one of the elements in terms of the mean longitudes and the elements of the orbits of the disturbed and the several disturbing bodies. If this rate of variation were given in terms of the time and known quantities, we should at once find the value of the element for any given time by a simple integration. But this is not the case.

The method of variation of elements gives us not a solution, but merely a transformation of our original differential equations of motion. The rates of variation are given in terms of the unknown elements themselves; and in order to find the elements from the equations so formed, we must employ repeated approximations.

Let us consider this matter a little more particularly.

The terms which express the rate of variation of any element may be divided into two classes-

I. Those which involve the mean longitudes of one or both of the planets concerned, as well as the elements of their orbits.

2. Those which involve the elements only.

The first are called periodic terms, since they pass from positive to negative, and vice vers $\hat{a}$, in periods comparable with those of the planets themselves. The second are called secular terms, and vary very slowly, since the elements on which they depend do so. Each of the terms in the expression of the rate of variation of any element will involve the mass of one of the disturbing bodies as a factor. Hence, if all these masses be very small, all the periodic inequalities of the elements will be likewise very small, and we shall obtain a value of the rate of variation which is very near the truth if we substitute for the complete value of any element its value when cleared of periodic inequalities. Then the periodic inequalities in 
the element under consideration may be found by direct integration, supposing the elements to be constant in the terms to be integrated, and the mean longitudes only to vary. Also the secular variation of the element considered, that is, the rate of variation of the element when cleared of periodic inequalities, will be given by the secular terms taken alone. If the disturbing masses, however, are not very small, this process is not sufficiently accurate, and the periodic inequalities thus found can only be regarded as a first approximation to the true values. In order to find more correct values, we must substitute for the elements in the second member of the equation their secular parts augmented by the approximate periodic inequalities before found.

Now, if in any periodic term we increase any element by a periodic inequality depending on a different argument, that is, involving different multiples of the mean longitudes, the result will evidently be to introduce new periodic terms which will involve the square of one of the masses or the product of two of them as a factor. Similarly, if in any periodic term any element be increased by a periodic inequality depending on the same argument, the result will also introduce new terms of the second order which do not involve the mean longitudes, and which therefore constitute new secular terms. These will be particularly important if the inequality in question be one of long period. Also in the secular terms the result of increasing any element by a periodic inequality will be to introduce a new periodic term depending on the same argument. Lastly, it should be remarked that in finding the periodic inequalities of any element by integration of the corresponding differential equation, we must take into account the secular variations of the elements which were neglected in the first approximation. The new terms thus introduced, like the others which we have just described, will evidently be of the second order with respect to the masses.

If the disturbing masses be large, as in the case of the mutual disturbances of Jupiter and Saturn, it may be necessary to proceed to a further approximation, and thus to obtain new terms, both periodic and secular, which involve the cubes and products of three dimensions of the masses. The number of combinations of terms which give rise to these terms of the second and third orders is practically unlimited, and the art of the calculator consists in selecting those combinations only which lead to sensible results. This is the chief cause of the great complexity of the theories of the larger planets, and more especially of those of Jupiter and Saturn.

M. Leverrier lays it down as the indispensable condition of all progress that we should be able to compare the whole of the observations of a planet with one and the same theory, however great may be the length of time over which the observations extend. In order to satisfy this condition, he develops the whole of his formulæ algebraically, leaving in a general symbolical form all the elements which vary with the time, such as the excentricities, the inclinations, and the longitudes of the perihelia and nodes. He treats in the same way the masses which are not yet sufficiently known.

All the work is given in full detail, and is divided as far as possible into parts independent of each other, so that any part may be readily verified. All the terms which are taken into account are clearly defined, so that if it should ever be necessary to carry on the approximations still further, it will be easy to do so without having to begin the investigation afresh. The whole work is presented with such clearness and method as to make it an admirable model for ail similar researches.

After the development of the disturbing functions, and the formation of the differential equations on which the variations of the elements depend, the first step to be taken is to determine by integration of these equations the periodic inequalities of the elements of the orbits of
Jupiter and Saturn which are of the first order with respect to the masses. As we have already said, the expressions of these periodic variations of the elements are given with such generality that, in order to obtain their numerical values at any epoch whatever, it is sufficient to substitute the secular values of the elements at that epoch. The calculation of the various terms under this general form is very laborious, and it requires great and sustained attention in order to avoid any error or omission of importance. On the other hand, by substituting from the beginning the numerical values of the elements at a given epoch the calculation is rendered much shorter and admits much more readily of verification; but the result thus obtained only holds good for the given epoch, and is thus entirely wanting in generality.

In the determination of the long inequalities of Jupiter and Saturn, the approximation is carried to terms which are of the seventh degree with respect to the excentricities and the mutual inclination of the orbits. In the next place the terms of the first order in the secular variations of the elements of the orbits are determined. After this the periodic inequalities of the second order with respect to the masses are considered. These are determined in the same form as the terms of the first order, in order that their expressions may hold good for any epoch whatever. The formulæ relating to these terms are necessarily very complicated. The coefficient belonging to a given argument depends, in general, on a great number of terms which are classed methodically. Next are determined the terms of the second order in the secular variations of the elements of the orbits. Afterwards, M. Leverrier takes into account the influence of the secular inequali ties on the values of the integrals on which the periodic inequalities depend. The last part of this chapter is devoted to the completion of the differential expressions of the secular inequalities by the determination of certain secular terms in the rates of variation of the excentricities and the longitudes of the perihelia, which are of the third and fourth orders with respect to the masses.

(To be continued.)

\section{NOTES}

WE record with sincere regret the death of Prof. Alphonse Oppenheim, at Hastings, on the 17 th inst.; he died by his own hand through grief at the death of his wife. Prof. Oppenheim is well known for his numerous researches in organic chemistry. Formerly one of the professors of chemistry at the University of Berlin, he only a few months ago, as we recorded at the time, had accepted the chair of chemistry at the University of Murster, in Westphalia. Prof. Oppenheim was a frequent contributor to this journal, and was much esteemed by a large circle of friends in England.

THE death is announced," on the 17 th inst., at the age of seventy-seven, of Mr. W. H. Fox Talbot, F.R.S., the inventor of the photographic process known as Talbotype, a name latterly merged in the general name photography. Mr. Talbot was a man of varied attainments and manifold work. He was educated at Harrow and Cambridge, where he distinguished himself as a Greek scholar. He took a delight in chemistry, and it was in I 833 that he seems to have conceived the idea of inventing some process by which the beautiful pictures exhibited in a camera lucida could be impressed and rendered permanent. He and Daguerre seem to have brought their several processes to a satisfactory result almost simultaneously, though Daguerre was the first to announce his process, in 1839 . Mr. Talbot lost no time in communicating to the Royal Society the details of his own process, though it was not till 1840 that he made the discovery which "laid the foundation of the photographic art in its present form." In I $842 \mathrm{Mr}$. Talbot was presented with the gold medal of the Royal Society. He did not patent his discovery, but on 